Original Article

\title{
Effects of purposeful action observation on kinematic patterns of upper extremity in individuals with hemiplegia
}

\author{
EunJOO KIM ${ }^{1)}$, KYEONGMi KIM ${ }^{2}{ }^{*}$ \\ 1) Department of Rehabilitation Science, Graduate School of Inje University, Republic of Korea \\ 2) Department of Occupational Therapy, College of Biomedical Sciences and Engineering, Inje \\ University: 197 Inje-ro, Gimhae-si, Gyeongsangnam-do 621-749, Republic of Korea
}

\begin{abstract}
Purpose] This study investigated the effects of purposeful action observation on upper extremity kinematic patterns in individuals with hemiplegia. [Subjects and Methods] Twelve patients were recruited in accordance with the inclusion criteria. The experimental group $(n=6)$ was trained with a purposeful action observation program. The control group $(n=6)$ was trained with only purposeful action without action observation. The programs were performed $30 \mathrm{~min} / \mathrm{session}, 5$ times per week for 30 sessions in 6 weeks of training. Upper extremity kinematic patterns were measured by a 3-dimensional motion analysis system before and after training, and the results were analyzed. [Results] The experimental group and the control group showed improvements in average velocity, trajectory ratio, and movement degree, but no statistically significant differences were observed between the groups. The experimental group showed statistically significant improvements in average velocity, trajectory ratio after the intervention. The experimental group also showed an improvement in movement degree, but the post-intervention difference was not significant. [Conclusion] The results of this study show that purposeful action observation training program improved the average velocity and trajectory ratio of stroke patients. Further research should enroll more subjects divided into more specific groups for treatment.

Key words: Action observation, Kinematic patterns, Stroke
\end{abstract}

(This article was submitted Jan. 16, 2015, and was accepted Feb. 26, 2015)

\section{INTRODUCTION}

Stroke is one of the three largest causes of death. Fiftythree out of 100,000 people die of stroke, and people older than 65 have a higher stroke rate ${ }^{1)}$. The number of stroke victims in 2030 is expected to be 3 times higher than that of today ${ }^{2}$. Neurological disorders including motor disorder, sensory disorder, cognitive disorder, perceptual disorder, speech disorder, and affective disorders may occur after a stroke. Motor disorders occur in about $80 \%$ of stroke patients and more than half of stroke patients suffer restriction of upper and lower extremity movement of the affected side due to hemiparesis ${ }^{3)}$. Especially, restriction of the upper extremity movement causes difficulty with maintaining an independent daily life. A recent development in the treatment and prognosis of upper limb function restoration is action observation training ${ }^{4)}$

Action observation training is based on the mirror neuron system theory which asserts that the same neurological

*Corresponding author. KyeongMi Kim (E-mail: kmik321@ inje.ac.kr)

C 2015 The Society of Physical Therapy Science. Published by IPEC Inc. This is an open-access article distributed under the terms of the Creative Commons Attribution Non-Commercial No Derivatives (by-ncnd) License $<$ http://creativecommons.org/licenses/by-nc-nd/3.0/>. changes occur when observing an action without actual performance as when actually performing the action. The mirror neuron system has been reported to be more activated when observing purposeful action than when observing simple action, and action observation training is more effective when a program uses assignments related to daily living activities $^{5}$. Buccino et al. ${ }^{6}$ studied neurologic changes elicited by action observation training. By showing that same area of the brain is activated in both situations they demonstrated that the same nervous system is used when observing an action as when performing it. Jong Man Kim et al. ${ }^{7}$ reported increases in the excitability of the corticospinal cord, as measured by the amplitude of movement evoked potentials during action observation training.

Porro et al. ${ }^{8)}$ reported functional change is an effect of action. They found that finger abduction increased after observing abduction action using the index and middle fingers of the right hand. Tae Woong Ha et al. ${ }^{9}$ reported that daily living activities and upper extremity function improved through observing the actions of holding a ball, a can, and a cup with a handle, and turning a faucet. Jin Woo Jo et al. ${ }^{10)}$ reported the effect of purposeful action observation on upper extremity function and promptness.

This study conducted a purposeful action observation program for stroke patients to identify the effect of the purposeful action observation program by measuring the kinematic patterns of the upper extremity before and after 
Table 1. Comparison of upper extremity changes in kinematic patterns of the groups and the pre-and post-intervention values of the experimental group

\begin{tabular}{lcccc}
\hline & \multicolumn{2}{c}{ Between groups } & \multicolumn{2}{c}{ Experimental group } \\
\cline { 2 - 5 } & Experimental group & Control group & Before & After \\
\hline Average velocity $(\mathrm{mm} / \mathrm{s})$ & $59.00 \pm 63.26$ & $12.83 \pm 310.766$ & $270.33 \pm 94.41$ & $329.33 \pm 125.1^{*}$ \\
Trajectory ratio & $-75.75 \pm 157.23$ & $-70.24 \pm 148.51$ & $81.07 \pm 163.7$ & $5.32 \pm 6.89^{*}$ \\
Motion angle $\left({ }^{\circ}\right)$ & $8.0 \pm 19.51$ & $18.66 \pm 35.41$ & $51.16 \pm 16.11$ & $59.16 \pm 14.38$ \\
\hline${ }^{*} \mathrm{p}<0.05$ & & & &
\end{tabular}

the program. This study also aimed to recommend purposeful action observation program as a mediating strategy for occupational treatment based on the results.

\section{SUBJECTS AND METHODS}

This study selected 12 study subjects from among patients who were diagnosed as having hemiplegia due to stroke. The subjects understood the purpose of this study and agreed to participate in the study. A nonequivalent pretestposttest control group design was used. The subjects were randomly assigned to the experimental group $(\mathrm{n}=6)$ and the control group $(n=6)$. The experimental group subjects received traditional occupational treatment and also performed a purposeful action observation training program. The control group subjects received traditional occupational treatment and topological treatment in which they performed purposeful action observation program assignments without actually observing the purposeful actions. The interventions were performed for 30 minutes per session, 5 sessions per week for 6 weeks, a total of 30 sessions.

A 3-dimensional motion analysis system was used to measure the changes in kinematic patterns of movement of the upper extremity. Winam software of Zebris Medical $\mathrm{GimbH}$, was used to analyze the motion of the arms of the affected side in 3-dimensions. The experimental equipment consisted of a measurement system for recording the movement, the Campact Measuring System10, a holding device, body surface markers, pads, cables, targets, and a computer. The study subjects were asked to sit on a chair located $10 \mathrm{~cm}$ away from their desk, trunk on the backrest move their hands to the starting point which was marked with tape, $5 \mathrm{~cm}$ from the edge of the desk, and adopt a posture of elbow flexion of $90^{\circ}$.

Three markers were attached to analyze the aiming motion of the upper extremity (average velocity and trajectory ratio). The markers were attached to the side of the index finger proximal interphalangeal and metacarpophalangeal joints, and the wrist. Markers for measuring the range of motion (motion angle) were attached to the dorsal side of the wrist in the middle, the elbow lateral epicondyle, and the insertion of the deltoid muscle.

The intervention period of the purposeful action observation program was selected based on the study of Feys et al. ${ }^{11)}$ : 30 minutes a day, 5 sessions a week for 6 weeks. Previous studies were reviewed for the design of assignments for purposeful action observation. The validity of the program was confirmed by 1 occupational treatment professor and 3 professional occupational therapists with more than 5 years of clinical experience. The purposeful action observation program is composed of assignment composition, selection of the difficulty levels, and methods.

The analysis of the data was performed using SPSS V18.0. The general characteristics of the study subjects are reported using descriptive statistics. The Mann Whitney U test was used to compare the results of the experimental and control groups. Wilcoxon's signed rank test was used to compare the upper extremity function of the affected side and kinematic patterns (average velocity, trajectory ratio, and motion (angle). A statistical significance level of 0.05 was used for all analyses.

\section{RESULTS}

The results of comparison of the kinematic patterns of the two groups are shown in Table 1. The post-intervention change in average velocity was $59.00 \pm 63.26 \mathrm{~mm} / \mathrm{s}$, that of the trajectory ratio was $-75.75 \pm 157.23$, and that of the motion angle was $8.0 \pm 19.51$ degrees in the experimental group. The post-intervention change in average velocity was $12.83 \pm 310.766 \mathrm{~mm} / \mathrm{s}$, that of the trajectory ratio was $-70.24 \pm 148.51$, and that of the motion angle was $18.66 \pm 35.41$ degrees in the control group.

The experimental group showed more improvement than to the control group, but the difference was not statistically significant $(p>0.05)$. Table 1 shows a comparison of the changes in the kinematic patterns of the groups, and pre-and post-intervention kinematic pattern values of the experimental group. All the changes in the kinematic patterns of the experimental group showed statistically significant improvements except that of motion angle.

\section{DISCUSSION}

The purpose of stroke rehabilitation is to reacquire the previously acquired skills. Action imitation is often used as the intervention method in rehabilitation. Action observation training is an action imitation method ${ }^{12}$.

The theoretical basis for action observation training is the activity of the mirror neuron system (MNS), and it utilizes observation of the actions of other people or a model shown in a video. The observed action activates the same nervous structure as the one activated when performing the actual $\operatorname{action}^{13)}$.

Previous studies which have used purposeful action observation program utilized simple repetition assignments 
for the training. However, the present study designed assignments imitating daily life related activities to support the assertion that action observation training is more effective when purposeful actions are observed rather than a simple action, and that assignments related to daily life change the functional effects of the training ${ }^{14}$.

The level of the assignment was considered in the selection of the purposeful action observation program assignments and patients were motivated by selecting patientoriented assignments ${ }^{15}$ ). Two assignments were picked for each session and repeated over one week. This was identical to the method of the study conducted by Feys et al., who suggested an intervention of 30 minutes a session, 5 sessions a week for 6 weeks. When a patient couldn't perform a difficult assignment, two assignments were repeatedly performed during the 6 weeks; thus, there was a possibility of lack of motivation and concentration in performance of the assignments.

The displacement and time from the ready position to the target was measured, and the average velocity was calculated using the two measurements. Therefore, higher average velocity means improved reactivity ${ }^{16)}$. The ratio of the distance actually moved to actual distance from the ready position to the target was calculated, and a ratio close to 1 implies high motion efficiency ${ }^{17}$ ). In other words, the statistically significant improvements in average velocity and trajectory ratio mean that the reactivity and efficiency of motion improved.

The strengths of this study are the inspection of validity by professionals, the design of the daily life related assignments of the action observation program through a review of previous studies, and patient motivation as the patients were given freedom to choose the assignments. Also, this study used a motion analysis system to objectively measure the upper extremity motions and kinematic patterns before and after the intervention, and analyzed the improvement in motion efficiency and velocity.

A limitation of this study was that it is hard to generalize the results of the study since the number of study subjects was small. Also, the measurements were taken only before and after the program; therefore, this study did not provide any information about the duration of the effects on upper extremity function after the intervention.

Future studies must investigate purposeful action observation programs with many more subjects to generalize the results. Follow-up studies are also needed to find out the post-intervention duration of improved kinematic patterns. Furthermore, future studies must pay attention to not only the upper extremity functions of stroke patients but also the efficiency, angle, and reactivity of the motion.

\section{REFERENCES}

1) National Statistical Office: Statistics for Causes of Death in. http://www. nso.go.kr.

2) World Health Organization: The top ten causes of death Geneva. Geneva: World Health Organization, 2007.

3) Urton ML, Kohia M, Davis J, et al.: Systematic literature review of treatment interventions for upper extremity hemiparesis following stroke. Occup Ther Int, 2007, 14: 11-27. [Medline] [CrossRef]

4) Woodson A: Occupational therapy for physical dysfunction, 4th ed. London: Williams \& Wilkins, 1995, pp 1300-1302.

5) Fogassi L, Ferrari PF, Gesierich B, et al.: Parietal lobe: from action organization to intention understanding. Science, 2005, 308: 662-667. [Medline] [CrossRef]

6) Buccino G, Binkofski F, Fink GR, et al.: Action observation activates premotor and parietal areas in a somatotopic manner: an fMRI study. Eur $\mathrm{J}$ Neurosci, 2001, 13: 400-404. [Medline]

7) Kim JM, Yang BI, Lee MK: The effect of action observational physical training on manual dexterity in stroke patients. Korean Research Society of Physical Therapy, 2010, 17: 17-24.

8) Porro CA, Facchin P, Fusi S, et al.: Enhancement of force after action observation: behavioural and neurophysiological studies. Neuropsychologia, 2007, 45: 3114-3121. [Medline] [CrossRef]

9) Tae UH: Effect of action observation training using motion picture on upper extremity unctions and activities of daily living of chronic stroke patient. Academic thesis. Department of rehabilitation Science Graduate School of Daegu University Rehabilitation, 2011.

10) Jin-Woo J, Soon BY, Lee MK: The effect of action observation training on upper extremity function in chronic stroke patients. J Korean Soc Occup Ther, 2011, 19: 15-24.

11) Feys HM, De Weerdt WJ, Selz BE, et al.: Effect of a therapeutic intervention for the hemiplegic upper limb in the acute phase after stroke: a singleblind, randomized, controlled multicenter trial. Stroke, 1998, 29: 785-792. [Medline] [CrossRef]

12) Buccino G, Solodkin A, Small SL: Functions of the mirror neuron system: implications for neurorehabilitation. Cogn Behav Neurol, 2006, 19: 55-63. [Medline] [CrossRef]

13) Small SL, Buccino G, Solodkin A: The mirror neuron system and treatment of stroke. Dev Psychobiol, 2012, 54: 293-310. [Medline] [CrossRef]

14) Horak, FB: Assumptions underlying motor control for neurologic rehabilitation, contemporary management of motor control problems: proceedings of IISTEP conference. Foundation of physical therapy, 1991.

15) Tickle-Degnen L: Client-centered practice, therapeutic relationship, and the use of research evidence. Am J Occup Ther, 2002, 56: 470-474. [Medline] [CrossRef]

16) Min-Ye J, Hyeseon J, Seok PH, et al.: The effect of constraint-induced movement therapy on persons with stroke evaluated by using 3D motion analysis. J Korean Soc Occup Ther, 2007, 15: 67-80.

17) Jung NH, Kim KM, Oh JS, et al.: The effects of bilateral arm task training on reaching performance and activities of daily living of stroke patients. $J$ Phys Ther Sci, 2013, 25: 449-452. [CrossRef] 